Proyecciones Journal of Mathematics

Vol. 34, No 4, pp. 323-341, December 2015.

Universidad Católica del Norte

Antofagasta - Chile

\title{
Inequalities of Hermite-Hadamard Type for $h$-Convex Functions on Linear Spaces
}

\author{
S. S. Dragomir \\ University of the Witwatersrand, South Africa \\ Received: March 2015. Accepted: September 2015
}

\begin{abstract}
Some inequalities of Hermite-Hadamard type for h-convex functions defined on convex subsets in real or complex linear spaces are given. Applications for norm inequalities are provided as well.
\end{abstract}

Subjclass : 26D15; 25D10.

Keywords : Convex functions, Integral inequalities, $h$-Convex functions. 


\section{Introduction}

The following inequality holds for any convex function $f$ defined on $\mathbf{R}$

$(1.1)(b-a) f\left(\frac{a+b}{2}\right)<\int_{a}^{b} f(x) d x<(b-a) \frac{f(a)+f(b)}{2}, \quad a, b \in \mathbf{R}$.

It was firstly discovered by Ch. Hermite in 1881 in the journal Mathesis (see [42]). But this result was nowhere mentioned in the mathematical literature and was not widely known as Hermite's result.

E. F. Beckenbach, a leading expert on the history and the theory of convex functions, wrote that this inequality was proven by J. Hadamard in 1893 [5]. In 1974, D. S. Mitrinović found Hermite's note in Mathesis [42]. Since (1.1) was known as Hadamard's inequality, the inequality is now commonly referred as the Hermite-Hadamard inequality.

For related results, see [10]-[19], [22]-[24], [31]-[34] and [45].

Let $X$ be a vector space over the real or complex number field $\mathbf{K}$ and $x, y \in X, x \neq y$. Define the segment

$$
[x, y]:=\{(1-t) x+t y, t \in[0,1]\} .
$$

We consider the function $f:[x, y] \rightarrow \mathbf{R}$ and the associated function

$$
g(x, y):[0,1] \rightarrow \mathbf{R}, g(x, y)(t):=f[(1-t) x+t y], t \in[0,1] .
$$

Note that $f$ is convex on $[x, y]$ if and only if $g(x, y)$ is convex on $[0,1]$.

For any convex function defined on a segment $[x, y] \subset X$, we have the Hermite-Hadamard integral inequality (see [20, p. 2], [21, p. 2])

$$
f\left(\frac{x+y}{2}\right) \leq \int_{0}^{1} f[(1-t) x+t y] d t \leq \frac{f(x)+f(y)}{2},
$$

which can be derived from the classical Hermite-Hadamard inequality (1.1) for the convex function $g(x, y):[0,1] \rightarrow \mathbf{R}$.

Since $f(x)=\|x\|^{p}(x \in X$ and $1 \leq p<\infty)$ is a convex function, then for any $x, y \in X$ we have the following norm inequality from (1.2) (see [45, p. 106])

$$
\left\|\frac{x+y}{2}\right\|^{p} \leq \int_{0}^{1}\|(1-t) x+t y\|^{p} d t \leq \frac{\|x\|^{p}+\|y\|^{p}}{2} .
$$


Motivated by the above results, in this paper we extend the concept of $h$-convexity introduced for functions of a real variable in [52] to functions defined on convex subsets of real or complex linear spaces and provide some Hermite-Hadamard type inequalities generalizing and improving (1.2). Natural applications that refine the norm inequality (1.3) are also given.

\section{2. $h$-Convex Functions on Linear Spaces}

We recall here some concepts of convexity that are well known in the literature. Let $I$ be an interval in $\mathbf{R}$.

Definition 1 ([36]). We say that $f: I \rightarrow \mathbf{R}$ is a Godunova-Levin function or that $f$ belongs to the class $Q(I)$ if $f$ is non-negative and for all $x, y \in I$ and $t \in(0,1)$ we have

$$
f(t x+(1-t) y) \leq \frac{1}{t} f(x)+\frac{1}{1-t} f(y) .
$$

Some further properties of this class of functions can be found in [27], [28], [30], [43], [46] and [47]. Among others, its has been noted that nonnegative monotone and non-negative convex functions belong to this class of functions.

The above concept can be extended for functions $f: C \subseteq X \rightarrow[0, \infty)$ where $C$ is a convex subset of the real or complex linear space $X$ and the inequality (2.1) is satisfied for any vectors $x, y \in C$ and $t \in(0,1)$. If the function $f: C \subseteq X \rightarrow \mathbf{R}$ is non-negative and convex, then is of GodunovaLevin type.

Definition 2 ([30]). We say that a function $f: I \rightarrow \mathbf{R}$ belongs to the class $P(I)$ if it is nonnegative and for all $x, y \in I$ and $t \in[0,1]$ we have

$$
f(t x+(1-t) y) \leq f(x)+f(y) .
$$

Obviously $Q(I)$ contains $P(I)$ and for applications it is important to note that also $P(I)$ contain all nonnegative monotone, convex and quasi convex functions, i. e. nonnegative functions satisfying

$$
f(t x+(1-t) y) \leq \max \{f(x), f(y)\}
$$

for all $x, y \in I$ and $t \in[0,1]$. 
For some results on $P$-functions see [30] and [44] while for quasi convex functions, the reader can consult [29].

If $f: C \subseteq X \rightarrow[0, \infty)$, where $C$ is a convex subset of the real or complex linear space $X$, then we say that it is of $P$-type (or quasi-convex) if the inequality (2.2) (or (2.3) holds true for $x, y \in C$ and $t \in[0,1]$.

Definition 3 ([7]). Let $s$ be a real number, $s \in(0,1]$. A function $f$ : $[0, \infty) \rightarrow[0, \infty)$ is said to be s-convex (in the second sense) or Breckner $s$-convex if

$$
f(t x+(1-t) y) \leq t^{s} f(x)+(1-t)^{s} f(y)
$$

for all $x, y \in[0, \infty)$ and $t \in[0,1]$.

For some properties of this class of functions see [1], [2], [7], [8], [25], [26], [37], [39] and [49].

The concept of Breckner $s$-convexity can be similarly extended for functions defined on convex subsets of linear spaces.

It is well known that if $(X,\|\cdot\|)$ is a normed linear space, then the function $f(x)=\|x\|^{p}, p \geq 1$ is convex on $X$.

Utilising the elementary inequality $(a+b)^{s} \leq a^{s}+b^{s}$ that holds for any $a, b \geq 0$ and $s \in(0,1]$, we have for the function $g(x)=\|x\|^{s}$ that

$$
\begin{aligned}
g(t x+(1-t) y) & =\|t x+(1-t) y\|^{s} \leq(t\|x\|+(1-t)\|y\|)^{s} \\
& \leq(t\|x\|)^{s}+[(1-t)\|y\|]^{s} \\
& =t^{s} g(x)+(1-t)^{s} g(y)
\end{aligned}
$$

In order to unify the above concepts for functions of real variable, S. Varošanec introduced the concept of $h$-convex functions as follows.

Assume that $I$ and $J$ are intervals in $\mathbf{R},(0,1) \subseteq J$ and functions $h$ and $f$ are real non-negative functions defined in $J$ and $I$, respectively.

Definition 4 ([52]). Let $h: J \rightarrow[0, \infty)$ with $h$ not identical to 0 . We say that $f: I \rightarrow[0, \infty)$ is an $h$-convex function if for all $x, y \in I$ we have

$$
f(t x+(1-t) y) \leq h(t) f(x)+h(1-t) f(y)
$$

for all $t \in(0,1)$. 
For some results concerning this class of functions see [52], [6], [40], [50], [48] and [51].

This concept can be extended for functions defined on convex subsets of linear spaces in the same way as above replacing the interval $I$ be the corresponding convex subset $C$ of the linear space $X$.

We can introduce now another class of functions.

Definition 5. We say that the function $f: C \subseteq X \rightarrow[0, \infty)$ is of $s$ Godunova-Levin type, with $s \in[0,1]$, if

$$
f(t x+(1-t) y) \leq \frac{1}{t^{s}} f(x)+\frac{1}{(1-t)^{s}} f(y),
$$

for all $t \in(0,1)$ and $x, y \in C$.

We observe that for $s=0$ we obtain the class of $P$-functions while for $s=1$ we obtain the class of Godunova-Levin. If we denote by $Q_{s}(C)$ the class of $s$-Godunova-Levin functions defined on $C$, then we obviously have

$$
\mathrm{P}(C)=Q_{0}(C) \subseteq Q_{s_{1}}(C) \subseteq Q_{s_{2}}(C) \subseteq Q_{1}(C)=Q(C)
$$
for $0 \leq s_{1} \leq s_{2} \leq 1$.

The cases of equality for various classes of convex functions have been studied by G. Maksa and Z. Páles in [43], who introduced the concept of affine functions and gave some characterizations of these functions. However, we do not present the details here. As observed by the referee, we only mention the fact that these functions can be used to prove the sharpness of some of the integral inequalities below.

We can prove now the following generalization of the Hermite-Hadamard inequality for $h$-convex functions defined on convex subsets of linear spaces.

Theorem 1. Assume that the function $f: C \subseteq X \rightarrow[0, \infty)$ is an $h$-convex function with $h \in L[0,1]$. Let $y, x \in C$ with $y \neq x$ and assume that the mapping $[0,1] \ni t \mapsto f[(1-t) x+t y]$ is Lebesgue integrable on $[0,1]$. Then

$$
\frac{1}{2 h\left(\frac{1}{2}\right)} f\left(\frac{x+y}{2}\right) \leq \int_{0}^{1} f[(1-t) x+t y] d t \leq[f(x)+f(y)] \int_{0}^{1} h(t) d t .
$$


Proof. By the $h$-convexity of $f$ we have

$$
f(t x+(1-t) y) \leq h(t) f(x)+h(1-t) f(y)
$$

for any $t \in[0,1]$.

Integrating $(2.7)$ on $[0,1]$ over $t$, we get

$$
\int_{0}^{1} f(t x+(1-t) y) d t \leq f(x) \int_{0}^{1} h(t) d t+f(y) \int_{0}^{1} h(1-t) d t
$$

and since $\int_{0}^{1} h(t) d t=\int_{0}^{1} h(1-t) d t$, we get the second part of $(2.6)$.

From the $h$-convexity of $f$ we have

$$
f\left(\frac{z+w}{2}\right) \leq h\left(\frac{1}{2}\right)[f(z)+f(w)]
$$

for any $z, w \in C$.

If we take in $(2.8) z=t x+(1-t) y$ and $w=(1-t) x+t y$, then we get

$$
f\left(\frac{x+y}{2}\right) \leq h\left(\frac{1}{2}\right)[f(t x+(1-t) y)+f((1-t) x+t y)]
$$

for any $t \in[0,1]$.

Integrating $(2.9)$ on $[0,1]$ over $t$ and taking into account that

$$
\int_{0}^{1} f(t x+(1-t) y) d t=\int_{0}^{1} f((1-t) x+t y) d t
$$

we get the first inequality in (2.6).

Remark 1. If $f: I \rightarrow[0, \infty)$ is an $h$-convex function on an interval $I$ of real numbers with $h \in L[0,1]$ and $f \in L[a, b]$ with $a, b \in I, a<b$, then from (2.6) we get the Hermite-Hadamard type inequality obtained by Sarikaya et al. in [48]

$$
\frac{1}{2 h\left(\frac{1}{2}\right)} f\left(\frac{a+b}{2}\right) \leq \int_{a}^{b} f(u) d u \leq[f(a)+f(b)] \int_{0}^{1} h(t) d t .
$$


If we write $(2.6)$ for $h(t)=t$, then we get the classical HermiteHadamard inequality for convex functions 1.2.

If we write (2.6) for the case of $P$-type functions $f: C \rightarrow[0, \infty)$, i.e., $h(t)=1, t \in[0,1]$, then we get the inequality

$$
\frac{1}{2} f\left(\frac{x+y}{2}\right) \leq \int_{0}^{1} f[(1-t) x+t y] d t \leq f(x)+f(y),
$$

that has been obtained for functions of real variable in [30].

If $f$ is Breckner $s$-convex on $C$, for $s \in(0,1)$, then by taking $h(t)=t^{s}$ in (2.6) we get

$$
2^{s-1} f\left(\frac{x+y}{2}\right) \leq \int_{0}^{1} f[(1-t) x+t y] d t \leq \frac{f(x)+f(y)}{s+1}
$$

that was obtained for functions of a real variable in [25].

Since the function $g(x)=\|x\|^{s}$ is Breckner $s$-convex on on the normed linear space $X, s \in(0,1)$, then for any $x, y \in X$ we have

$$
\frac{1}{2}\|x+y\|^{s} \leq \int_{0}^{1}\|(1-t) x+t y\|^{s} d t \leq \frac{\|x\|^{s}+\|x\|^{s}}{s+1} .
$$

If $f: C \rightarrow[0, \infty)$ is of $s$-Godunova-Levin type, with $s \in[0,1)$, then

$$
\frac{1}{2^{s+1}} f\left(\frac{x+y}{2}\right) \leq \int_{0}^{1} f[(1-t) x+t y] d t \leq \frac{f(x)+f(y)}{1-s} .
$$

We notice that for $s=1$ the first inequality in (2.13) still holds, i. e.

$$
\frac{1}{4} f\left(\frac{x+y}{2}\right) \leq \int_{0}^{1} f[(1-t) x+t y] d t
$$

The case for functions of real variables was obtained for the first time in [30].

\section{Refinements}

The following representation result holds.

Lemma 1. Let $f: C \subseteq X \rightarrow \mathbf{C}$ where $C$ is a convex subset of the real or complex linear space $X$. Let $y, x \in C$ with $y \neq x$ and assume that the mapping $[0,1] \ni t \mapsto f[(1-t) x+t y]$ is Lebesgue integrable on $[0,1]$. Then for any $\lambda \in[0,1]$ we have the representation 


$$
\begin{aligned}
& \int_{0}^{1} f[(1-t) x+t y] d t=(1-\lambda) \int_{0}^{1} f[(1-t)((1-\lambda) x+\lambda y)+t y] d t \\
& +\lambda \int_{0}^{1} f[(1-t) x+t((1-\lambda) x+\lambda y)] d t .
\end{aligned}
$$

Proof. For $\lambda=0$ and $\lambda=1$ the equality (3.1) is obvious.

Let $\lambda \in(0,1)$. Observe that

$$
\begin{gathered}
\int_{0}^{1} f[(1-t)(\lambda y+(1-\lambda) x)+t y] d t \\
=\int_{0}^{1} f[((1-t) \lambda+t) y+(1-t)(1-\lambda) x] d t
\end{gathered}
$$

and

$$
\int_{0}^{1} f[t(\lambda y+(1-\lambda) x)+(1-t) x] d t=\int_{0}^{1} f[t \lambda y+(1-\lambda t) x] d t .
$$

If we make the change of variable $u:=(1-t) \lambda+t$ then we have

$1-\mathrm{u}=(1-t)(1-\lambda)$ and $d u=(1-\lambda) d u$. Then

$$
\int_{0}^{1} f[((1-t) \lambda+t) y+(1-t)(1-\lambda) x] d t=\frac{1}{1-\lambda} \int_{\lambda}^{1} f[u y+(1-u) x] d u .
$$

If we make the change of variable $u:=\lambda t$ then we have $d u=\lambda d t$ and

$$
\int_{0}^{1} f[t \lambda y+(1-\lambda t) x] d t=\frac{1}{\lambda} \int_{0}^{\lambda} f[u y+(1-u) x] d u .
$$

Therefore

$$
\begin{gathered}
(1-\lambda) \int_{0}^{1} f[(1-t)(\lambda y+(1-\lambda) x)+t y] d t \\
+\lambda \int_{0}^{1} f[t(\lambda y+(1-\lambda) x)+(1-t) x] d t \\
=\int_{\lambda}^{1} f[u y+(1-u) x] d u+\int_{0}^{\lambda} f[u y+(1-u) x] d u
\end{gathered}
$$




$$
=\int_{0}^{1} f[u y+(1-u) x] d u
$$

and the identity (3.1) is proved.

Theorem 2. Assume that the function $f: C \subseteq X \rightarrow[0, \infty)$ is an $h$-convex function with $h \in L[0,1]$. Let $y, x \in C$ with $y \neq x$ and assume that the mapping $[0,1] \ni t \mapsto f[(1-t) x+t y]$ is Lebesgue integrable on $[0,1]$. Then for any $\lambda \in[0,1]$ we have the inequalities

$$
\begin{aligned}
& \frac{1}{2 h\left(\frac{1}{2}\right)}\left\{(1-\lambda) f\left[\frac{(1-\lambda) x+(\lambda+1) y}{2}\right]+\lambda f\left[\frac{(2-\lambda) x+\lambda y}{2}\right]\right\} \\
& \leq \int_{0}^{1} f[(1-t) x+t y] d t \\
& \leq[f((1-\lambda) x+\lambda y)+(1-\lambda) f(y)+\lambda f(x)] \int_{0}^{1} h(t) d t \\
& \leq\{[h(1-\lambda)+\lambda] f(x)+[h(\lambda)+1-\lambda] f(y)\} \int_{0}^{1} h(t) d t .
\end{aligned}
$$

Proof. Since $f: C \subseteq X \rightarrow[0, \infty)$ is an $h$-convex function, then by Theorem 1 we have

$$
\begin{aligned}
& \frac{1}{2 h\left(\frac{1}{2}\right)} f\left[\frac{(1-\lambda) x+(\lambda+1) y}{2}\right] \leq \int_{0}^{1} f[(1-t)((1-\lambda) x+\lambda y)+t y] d t \\
& \leq[f((1-\lambda) x+\lambda y)+f(y)] \int_{0}^{1} h(t) d t
\end{aligned}
$$

and

$$
\begin{aligned}
& \frac{1}{2 h\left(\frac{1}{2}\right)} f\left[\frac{(2-\lambda) x+\lambda y}{2}\right] \leq \int_{0}^{1} f[(1-t) x+t((1-\lambda) x+\lambda y)] d t \\
& \leq[f(x)+f((1-\lambda) x+\lambda y)] \int_{0}^{1} h(t) d t .
\end{aligned}
$$


Now, if we multiply the inequality (2.3) by $1-\lambda \geq 0$ and (3.4) by $\lambda \geq 0$ and add the obtained inequalities, then we get

$$
\begin{aligned}
& \frac{1-\lambda}{2 h\left(\frac{1}{2}\right)} f\left[\frac{(1-\lambda) x+(\lambda+1) y}{2}\right]+\frac{\lambda}{2 h\left(\frac{1}{2}\right)} f\left[\frac{(2-\lambda) x+\lambda y}{2}\right] \\
\leq & (1-\lambda) \int_{0}^{1} f[(1-t)((1-\lambda) x+\lambda y)+t y] d t \\
+ & \lambda \int_{0}^{1} f[(1-t) x+t((1-\lambda) x+\lambda y)] d t \\
\leq & (1-\lambda)[f((1-\lambda) x+\lambda y)+f(y)] \int_{0}^{1} h(t) d t \\
+ & \lambda[f(x)+f((1-\lambda) x+\lambda y)] \int_{0}^{1} h(t) d t
\end{aligned}
$$

and by (3.1) we obtain the first two inequalities in (3.2).

The last part is obvious.

Remark 2. With the assumptions from Theorem 2, we observe that if we take either $\lambda=0$ or $\lambda=1$ in the first two inequalities in (3.2), then we get (2.6).

If we take $\lambda=\frac{1}{2}$ and use the $h$-convexity of $f$, then we get from (3.2) that

$$
\begin{aligned}
& \frac{1}{4 h^{2}\left(\frac{1}{2}\right)} f\left(\frac{x+y}{2}\right) \leq \frac{1}{4 h\left(\frac{1}{2}\right)}\left\{f\left(\frac{x+3 y}{4}\right)+f\left(\frac{3 x+y}{4}\right)\right\} \\
\leq & \int_{0}^{1} f[(1-t) x+t y] d t \\
\leq & {\left[f\left(\frac{x+y}{2}\right)+\frac{f(x)+f(y)}{2}\right] \int_{0}^{1} h(t) d t } \\
\leq & {\left[h\left(\frac{1}{2}\right)+\frac{1}{2}\right][f(x)+f(y)] \int_{0}^{1} h(t) d t . }
\end{aligned}
$$


In general, if $h(\lambda)>0$ for $\lambda \in(0,1)$, then

$$
\begin{gathered}
(1-\lambda) f\left[\frac{(1-\lambda) x+(\lambda+1) y}{2}\right]+\lambda f\left[\frac{(2-\lambda) x+\lambda y}{2}\right] \\
=\frac{1-\lambda}{h(1-\lambda)} h(1-\lambda) f\left[\frac{(1-\lambda) x+(\lambda+1) y}{2}\right] \\
+\frac{\lambda}{h(\lambda)} h(\lambda) f\left[\frac{(2-\lambda) x+\lambda y}{2}\right] \\
\times\left\{\min \left\{\frac{1-\lambda}{h(1-\lambda)}, \frac{\lambda}{h(\lambda)}\right\}\right. \\
\left.\times\{1-\lambda) f\left[\frac{(1-\lambda) x+(\lambda+1) y}{2}\right]+h(\lambda) f\left[\frac{(2-\lambda) x+\lambda y}{2}\right]\right\} \\
\times f\left[(1-\lambda) \frac{(1-\lambda) x+(\lambda+1) y}{2}+\lambda \frac{(2-\lambda) x+\lambda y}{2}\right] \\
=\min \left\{\frac{1-\lambda}{h(1-\lambda)}, \frac{\lambda}{h(\lambda)}\right\} f\left(\frac{x+y}{2}\right)
\end{gathered}
$$

and from (3.2) we get the sequence of inequalities

$$
\begin{aligned}
& \frac{1}{2 h\left(\frac{1}{2}\right)} \min \left\{\frac{1-\lambda}{h(1-\lambda)}, \frac{\lambda}{h(\lambda)}\right\} f\left(\frac{x+y}{2}\right) \\
\leq & \frac{1}{2 h\left(\frac{1}{2}\right)}\left\{(1-\lambda) f\left[\frac{(1-\lambda) x+(\lambda+1) y}{2}\right]+\lambda f\left[\frac{(2-\lambda) x+\lambda y}{2}\right]\right\} \\
\leq & \int_{0}^{1} f[(1-t) x+t y] d t \\
\leq & {[f((1-\lambda) x+\lambda y)+(1-\lambda) f(y)+\lambda f(x)] \int_{0}^{1} h(t) d t } \\
\leq & \{[h(1-\lambda)+\lambda] f(x)+[h(\lambda)+1-\lambda] f(y)\} \int_{0}^{1} h(t) d t .
\end{aligned}
$$


Corollary 1. Let $f: C \subseteq X \rightarrow[0, \infty)$ be a convex function on the convex set $C$ in a linear space $X$. Then for any $y, x \in C$ and for any $\lambda \in[0,1]$ we have the inequalities

$$
\begin{aligned}
& f\left(\frac{x+y}{2}\right) \leq(1-\lambda) f\left[\frac{(1-\lambda) x+(\lambda+1) y}{2}\right]+\lambda f\left[\frac{(2-\lambda) x+\lambda y}{2}\right] \\
& \leq \int_{0}^{1} f[(1-t) x+t y] d t \\
& \leq \frac{1}{2}[f((1-\lambda) x+\lambda y)+(1-\lambda) f(y)+\lambda f(x)] \\
& \leq \frac{f(y)+f(x)}{2} .
\end{aligned}
$$

Remark 3. The inequality (3.8) has been obtained for functions of a real variable by $A$. El Farissi in [35].

The inequality (3.8) provides the following norm inequality:

$$
\begin{aligned}
& \left\|\frac{x+y}{2}\right\|^{p} \leq(1-\lambda)\left\|\frac{(1-\lambda) x+(\lambda+1) y}{2}\right\|^{p}+\lambda\left\|\frac{(2-\lambda) x+\lambda y}{2}\right\|^{p} \\
& \leq \int_{0}^{1}\|(1-t) x+t y\|^{p} d t \\
& \leq \frac{1}{2}\left[\|(1-\lambda) x+\lambda y\|^{p}+(1-\lambda)\|y\|^{p}+\lambda\|x\|^{p}\right] \leq \frac{\|y\|^{p}+\|x\|^{p}}{2},
\end{aligned}
$$

that holds for any $x, y \in X$, a normed space, $p \geq 1$ and $\lambda \in[0,1]$.

Corollary 2. Assume that the function $f: C \subseteq X \rightarrow[0, \infty)$ is a Breckner $s$-convex function with $s \in(0,1)$. Let $y, x \in C$ with $y \neq x$ and assume that the mapping $[0,1] \ni t \mapsto f[(1-t) x+t y]$ is Lebesgue integrable on $[0,1]$. Then for any $\lambda \in(0,1)$ we have the inequalities

$$
2^{s-1}\left(\frac{1}{2}-\left|\frac{1}{2}-\lambda\right|\right)^{1-s} f\left(\frac{x+y}{2}\right)
$$




$$
\begin{aligned}
& \leq 2^{s-1}\left\{(1-\lambda) f\left[\frac{(1-\lambda) x+(\lambda+1) y}{2}\right]+\lambda f\left[\frac{(2-\lambda) x+\lambda y}{2}\right]\right\} \\
& \leq \int_{0}^{1} f[(1-t) x+t y] d t \\
& \leq \frac{1}{s+1}[f((1-\lambda) x+\lambda y)+(1-\lambda) f(y)+\lambda f(x)] \\
& \leq \frac{1}{s+1}\left\{\left[(1-\lambda)^{s}+\lambda\right] f(x)+\left(\lambda^{s}+1-\lambda\right) f(y)\right\} .
\end{aligned}
$$

The inequality (3.10) provides the following norm inequality:

$$
\begin{aligned}
& \quad 2^{s-1}\left(\frac{1}{2}-\left|\frac{1}{2}-\lambda\right|\right)^{1-s}\left\|\frac{x+y}{2}\right\|^{s} \\
& \leq 2^{s-1}\left\{(1-\lambda)\left\|\frac{(1-\lambda) x+(\lambda+1) y}{2}\right\|^{s}+\lambda\left\|\frac{(2-\lambda) x+\lambda y}{2}\right\|^{s}\right\} \\
& \leq \int_{0}^{1}\|(1-t) x+t y\|^{s} d t \\
& \leq \frac{1}{s+1}\left[\|(1-\lambda) x+\lambda y\|^{s}+(1-\lambda)\|y\|^{s}+\lambda\|x\|^{s}\right] \\
& \leq \frac{1}{s+1}\left\{\left[(1-\lambda)^{s}+\lambda\right]\|x\|^{s}+\left(\lambda^{s}+1-\lambda\right)\|y\|^{s}\right\}
\end{aligned}
$$

that holds for any $x, y \in X$, a normed space, $s \in(0,1)$ and $\lambda \in(0,1)$.

In particular, we have

$$
\begin{aligned}
& 4^{s-1}\left\|\frac{x+y}{2}\right\|^{s} \leq 2^{s-2}\left\{\left\|\frac{x+3 y}{4}\right\|^{s}+\left\|\frac{3 x+y}{4}\right\|^{s}\right\} \\
& \leq \int_{0}^{1}\|(1-t) x+t y\|^{s} d t \\
& \leq \frac{1}{s+1}\left[\left\|\frac{x+y}{2}\right\|^{s}+\frac{\|y\|^{s}+\|x\|^{s}}{2}\right] \leq \frac{1+2^{1-s}}{(s+1) 2^{s}}\left(\|x\|^{s}+\|y\|^{s}\right),
\end{aligned}
$$

for any $x, y \in X$ and $s \in(0,1)$. 
Remark 4. Similar inequalities can be stated for functions of $s$-GodunovaLevin type, with $s \in[0,1)$, however the details are omitted.

Acknowledgement. The author would like to thank the anonymous referee for valuable comments that have been implemented in the final version of the paper.

\section{References}

[1] M. Alomari and M. Darus, The Hadamard's inequality for s-convex function. Int. J. Math. Anal. (Ruse) 2, No. 13-16, pp. 639-646, (2008).

[2] M. Alomari and M. Darus, Hadamard-type inequalities for s-convex functions. Int. Math. Forum 3, No. 37-40, pp. 1965-1975, (2008).

[3] G. A. Anastassiou, Univariate Ostrowski inequalities, revisited. Monatsh. Math., 135, No. 3, pp. 175-189, (2002).

[4] N. S. Barnett, P. Cerone, S. S. Dragomir, M. R. Pinheiro,and A. Sofo, Ostrowski type inequalities for functions whose modulus of the derivatives are convex and applications. Inequality Theory and Applications, Vol. 2 (Chinju/Masan, 2001), 19-32, Nova Sci. Publ., Hauppauge, NY, 2003. Preprint: RGMIA Res. Rep. Coll. 5 (2002), No. 2, Art. 1 [Online http://rgmia.org/papers/v5n2/Paperwapp2q.pdf].

[5] E. F. Beckenbach, Convex functions, Bull. Amer. Math. Soc. 54, pp. 439-460, (1948).

[6] M. Bombardelli and S. Varošanec, Properties of h-convex functions related to the Hermite-Hadamard-Fejér inequalities. Comput. Math. Appl. 58, No. 9, pp. 1869-1877, (2009).

[7] W. W. Breckner, Stetigkeitsaussagen für eine Klasse verallgemeinerter konvexer Funktionen in topologischen linearen Räumen. (German) Publ. Inst. Math. (Beograd) (N.S.) 23(37), pp. 13-20, (1978).

[8] W. W. Breckner and G. Orbán, Continuity properties of rationally s-convex mappings with values in an ordered topological linear space. Universitatea "Babeş-Bolyai, Facultatea de Matematica, Cluj-Napoca, (1978). viii+92. 
[9] P. Cerone and S. S. Dragomir, Midpoint-type rules from an inequalities point of view, Ed. G. A. Anastassiou, Handbook of AnalyticComputational Methods in Applied Mathematics, CRC Press, New York. 135-200.

[10] P. Cerone and S. S. Dragomir, New bounds for the three-point rule involving the Riemann-Stieltjes integrals, in Advances in Statistics Combinatorics and Related Areas, C. Gulati, et al. (Eds.), World Science Publishing, pp. 53-62, (2002).

[11] P. Cerone, S. S. Dragomir and J. Roumeliotis, Some Ostrowski type inequalities for $n$-time differentiable mappings and applications, Demonstratio Mathematica, 32(2), pp. 697-712, (1999).

[12] G. Cristescu, Hadamard type inequalities for convolution of $h$-convex functions. Ann. Tiberiu Popoviciu Semin. Funct. Equ. Approx. Convexity 8, pp. 3-11, (2010).

[13] S. S. Dragomir, Ostrowski's inequality for monotonous mappings and applications, J. KSIAM, 3(1), pp. 127-135, (1999).

[14] S. S. Dragomir, The Ostrowski's integral inequality for Lipschitzian mappings and applications, Comp. Math. Appl., 38, pp. 33-37, (1999).

[15] S. S. Dragomir, On the Ostrowski's inequality for Riemann-Stieltjes integral, Korean J. Appl. Math., 7, pp. 477-485, (2000).

[16] S. S. Dragomir, On the Ostrowski's inequality for mappings of bounded variation and applications, Math. Ineq. \& Appl., 4 (1), pp. 33-40, (2001).

[17] S. S. Dragomir, On the Ostrowski inequality for Riemann-Stieltjes integral $\int_{a}^{b} f(t) d u(t)$ where $f$ is of Hölder type and $u$ is of bounded variation and applications, J. KSIAM, 5 (1), pp. 35-45, (2001).

[18] S. S. Dragomir, Ostrowski type inequalities for isotonic linear functionals, J. Inequal. Pure E Appl. Math., 3(5) (2002), Art. 68.

[19] S. S. Dragomir, An inequality improving the first Hermite-Hadamard inequality for convex functions defined on linear spaces and applications for semi-inner products. J. Inequal. Pure Appl. Math. 3 (2002), no. 2, Article 31,8 pp. 
[20] S. S. Dragomir, An inequality improving the first Hermite-Hadamard inequality for convex functions defined on linear spaces and applications for semi-inner products, J. Inequal. Pure Appl. Math. 3 (2002), No. 2, Article 31.

[21] S. S. Dragomir, An inequality improving the second HermiteHadamard inequality for convex functions defined on linear spaces and applications for semi-inner products, J. Inequal. Pure Appl. Math. 3 (2002), No.3, Article 35.

[22] S. S. Dragomir, An Ostrowski like inequality for convex functions and applications, Revista Math. Complutense, 16 (2), pp. 373-382, (2003).

[23] S. S. Dragomir, Operator Inequalities of Ostrowski and Trapezoidal Type. Springer Briefs in Mathematics. Springer, New York, (2012). x+112 pp. ISBN: 978-1-4614-1778-1.

[24] S. S. Dragomir, P. Cerone, J. Roumeliotis and S. Wang, A weighted version of Ostrowski inequality for mappings of Hölder type and applications in numerical analysis, Bull. Math. Soc. Sci. Math. Romanie, 42 (90) (4), pp. 301-314, (1999).

[25] S.S. Dragomir and S. Fitzpatrick, The Hadamard inequalities for sconvex functions in the second sense. Demonstratio Math. 32, No. 4, pp. 687-696, (1999).

[26] S.S. Dragomir and S. Fitzpatrick, The Jensen inequality for s-Breckner convex functions in linear spaces. Demonstratio Math. 33, No. 1, pp. 43-49, (2000).

[27] S. S. Dragomir and B. Mond, On Hadamard's inequality for a class of functions of Godunova and Levin. Indian J. Math. 39, No. 1, pp. 1-9, (1997).

[28] S. S. Dragomir and C. E. M. Pearce, On Jensen's inequality for a class of functions of Godunova and Levin. Period. Math. Hungar. 33, No. 2, pp. 93-100, (1996).

[29] S. S. Dragomir and C. E. M. Pearce, Quasi-convex functions and Hadamard's inequality, Bull. Austral. Math. Soc. 57, pp. 377-385, (1998).

[30] S. S. Dragomir, J. Pečarić and L. Persson, Some inequalities of Hadamard type. Soochow J. Math. 21, No. 3, pp. 335-341, (1995). 
[31] S. S. Dragomir and Th. M. Rassias (Eds), Ostrowski Type Inequalities and Applications in Numerical Integration, Kluwer Academic Publisher, (2002).

[32] S. S. Dragomir and S. Wang, A new inequality of Ostrowski's type in $L_{1}$-norm and applications to some special means and to some numerical quadrature rules, Tamkang J. of Math., 28, pp. 239-244, (1997).

[33] S. S. Dragomir and S. Wang, Applications of Ostrowski's inequality to the estimation of error bounds for some special means and some numerical quadrature rules, Appl. Math. Lett., 11 , pp. 105-109, (1998).

[34] S. S. Dragomir and S. Wang, A new inequality of Ostrowski's type in $L_{p}$-norm and applications to some special means and to some numerical quadrature rules, Indian J. of Math., 40 (3), pp. 245-304, (1998).

[35] A. El Farissi, Simple proof and refeinment of Hermite-Hadamard inequality, J. Math. Ineq. 4, No. 3, pp. 365-369, (2010).

[36] E. K. Godunova and V. I. Levin, Inequalities for functions of a broad class that contains convex, monotone and some other forms of functions. (Russian) Numerical mathematics and mathematical physics (Russian), pp. 138-142, 166, Moskov. Gos. Ped. Inst., Moscow, (1985).

[37] H. Hudzik and L. Maligranda, Some remarks on s-convex functions. Aequationes Math. 48, No. 1, 100-111, (1994).

[38] E. Kikianty and S. S. Dragomir, Hermite-Hadamard's inequality and the p-HH-norm on the Cartesian product of two copies of a normed space, Math. Inequal. Appl. (in press)

[39] U. S. Kirmaci, M. Klaričić Bakula, M. E Özdemir and J. Pečarić, Hadamard-type inequalities for s-convex functions. Appl. Math. Comput. 193, No. 1, pp. 26-35, (2007).

[40] M. A. Latif, On some inequalities for h-convex functions. Int. J. Math. Anal. (Ruse) 4, No. 29-32, pp. 1473-1482, (2010).

[41] G. Maksa and Z. Páles, The equality case in some recent convexity inequalities, Opuscula Math. 31, 2, pp. 269-277, (2011).

[42] D. S. Mitrinović and I. B. Lacković, Hermite and convexity, Aequationes Math. 28, pp. 229-232, (1985). 
[43] D. S. Mitrinović and J. E. Pečarić, Note on a class of functions of Godunova and Levin. C. R. Math. Rep. Acad. Sci. Canada 12, No. 1, pp. 33-36, (1990).

[44] C. E. M. Pearce and A. M. Rubinov, P-functions, quasi-convex functions, and Hadamard-type inequalities. J. Math. Anal. Appl. 240, No. 1, pp. 92-104, (1999).

[45] J. E. Pečarić and S. S. Dragomir, On an inequality of Godunova-Levin and some refinements of Jensen integral inequality. Itinerant Seminar on Functional Equations, Approximation and Convexity (Cluj-Napoca, 1989), 263-268, Preprint, 89-6, Univ. "Babeş-Bolyai, Cluj-Napoca, (1989).

[46] J. Pečarić and S. S. Dragomir, A generalization of Hadamard's inequality for isotonic linear functionals, Radovi Mat. (Sarajevo) 7, pp. 103-107, (1991).

[47] M. Radulescu, S. Radulescu and P. Alexandrescu, On the GodunovaLevin-Schur class of functions. Math. Inequal. Appl. 12, No. 4, pp. 853-862, (2009).

[48] M. Z. Sarikaya, A. Saglam, and H. Yildirim, On some Hadamard-type inequalities for h-convex functions. J. Math. Inequal. 2, No. 3, pp. 335-341, (2008).

[49] E. Set, M. E. Özdemir and M. Z. Sarıkaya, New inequalities of Ostrowski's type for s-convex functions in the second sense with applications. Facta Univ. Ser. Math. Inform. 27, No. 1, pp. 67-82, (2012).

[50] M. Z. Sarikaya, E. Set and M. E. Özdemir, On some new inequalities of Hadamard type involving h-convex functions. Acta Math. Univ. Comenian. (N.S.) 79, pp. 265-272, No. 2, (2010).

[51] M. Tunç, Ostrowski-type inequalities via h-convex functions with applications to special means. J. Inequal. Appl., 2013, 2013:326.

[52] S. Varošanec, On h-convexity. J. Math. Anal. Appl. 326, No. 1, pp. 303-311, (2007). 


\section{S. S. Dragomir}

Mathematics,

College of Engineering \& Science

Victoria University,

P. O. Box 14428

Melbourne City,

MC 8001,

Australia

e-mail : sever.dragomir@vu.edu.au 